Case Report

\title{
Podocyturia: A Clue for the Rational Use of Amiloride in Alport Renal Disease
}

\author{
H. Trimarchi, ${ }^{1}$ R. Canzonieri, ${ }^{2}$ A. Muryan, ${ }^{2}$ A. Schiel, ${ }^{2}$ A. Araoz, ${ }^{3}$ M. Paulero, ${ }^{1}$ J. Andrews, ${ }^{1}$ \\ T. Rengel, ${ }^{1}$ M. Forrester, ${ }^{1}$ F. Lombi, ${ }^{1}$ V. Pomeranz, ${ }^{1}$ R. Iriarte, ${ }^{1}$ and E. Zotta ${ }^{3}$ \\ ${ }^{1}$ Nephrology Service, Hospital Britanico de Buenos Aires, Perdriel 74, 1280 Buenos Aires, Argentina \\ ${ }^{2}$ Laboratory Service, Hospital Britanico de Buenos Aires, Perdriel 74, 1280 Buenos Aires, Argentina \\ ${ }^{3}$ IFIBIO Houssay, UBA CONICET, Facultad de Medicina, Universidad de Buenos Aires, Paraguay 2155, 1425 Buenos Aires, Argentina
}

Correspondence should be addressed to H. Trimarchi; htrimarchi@hotmail.com

Received 11 October 2015; Accepted 24 December 2015

Academic Editor: Kandai Nozu

Copyright (C) 2016 H. Trimarchi et al. This is an open access article distributed under the Creative Commons Attribution License, which permits unrestricted use, distribution, and reproduction in any medium, provided the original work is properly cited.

\begin{abstract}
No specific or efficient treatment exists for Alport syndrome, an X-linked hereditary disease caused by mutations in collagen type IV, a crucial component of the glomerular basement membrane. Kidney failure is usually a major complication of the disease, and patients require renal replacement therapy early in life. Microhematuria and subsequently proteinuria are hallmarks of kidney involvement, which are due to primary basement membrane alterations that mainly cause endothelial thrombosis and podocyte contraction and ulterior irreversible detachment. Commonly drug-based approaches include angiotensin-converting enzyme inhibitors and angiotensin receptor blockers, which are employed to reduce proteinuria and thus retard kidney disease progression and cardiovascular morbidity and mortality. However, as any hereditary disease, it is expressed as early as in the intrauterine life, and usually an index case is helpful to detect family-related cases. As no specific treatment exists, pathophysiologically based approaches are useful. The present case illustrates the reduction rate of urinary podocyte loss and proteinuria after amiloride administration and suggests the molecular pathways involved in Alport renal disease. Finally, podocyturia rather than proteinuria should be considered as an earlier biomarker of kidney involvement and disease progression in Alport disease.
\end{abstract}

\section{Introduction}

Alport syndrome is a chromosome X-linked hereditary disease with systemic involvement, mainly affecting the renal, pulmonary, visual, and auditory systems. It is due to different mutations in collagen IV [1]. Alport disease lacks specific therapy. Aims are directed to reducing the rate of progression of the organs involved, whenever possible. In this respect, chronic kidney disease is managed with the usual nephroprotective recommendations, as loss of weight, blood pressure control, salt restriction, and tobacco avoidance, amongst others. Angiotensin-converting enzyme inhibitors and angiotensin receptor blockers have been shown to be helpful to reduce kidney function decline, particularly when proteinuria exists [2]. Regrettably, only relatives linked to the index case take profit of these limited recommendations. All patients progress to end-stage kidney disease early in adulthood [1]. Therefore, early effective interventions in asymptomatic patients are mandatory. Urinary podocyte loss is a silent phenomenon that precedes proteinuria in glomerular diseases $[3,4]$. Podocyturia is irreversible and any attempt to decrease its amount, particularly in early phases of a glomerulopathy, should be accompanied by reductions in proteinuria and delays in kidney function decline. We present a young male patient with a family history of kidney biopsyproven Alport disease. He had normal kidney function, microhematuria, and mild proteinuria. His podocyturia was higher than age-matched controls. After amiloride prescription, his proteinuria turned negative and was detected as microalbuminuria, while podocyturia decreased to control rates. We suggest the potential roles certain integrins, the urokinase plasminogen activator (uPA), its receptor (uPAR), and plasmin may play in the pathogenesis of Alport kidney disease. 


\section{Case Presentation}

A 25-year-old male with a family background of Alport disease was referred for an assessment of kidney involvement. Family history consisted of a mother-related uncle on dialysis with biopsy-proven Alport disease, another mother-related cousin with kidney involvement, and a grandfather who died due to sudden death at the age of 42 . The patient was asymptomatic and normotensive (blood pressure 115/68 $\mathrm{mmHg}$ ), with a body mass index of 24 and no tobacco consumption. Main laboratory results included hematocrit $44 \%$, glycemia $78 \mathrm{mg} / \mathrm{dL}$, serum creatinine $1.07 \mathrm{mg} / \mathrm{dL}$, creatinine clearance of $74 \mathrm{~mL} / \mathrm{min}$, sodium $143 \mathrm{mEq} / \mathrm{L}$, potassium $3.9 \mathrm{mEq} / \mathrm{L}$, serum albumin $4.3 \mathrm{~g} / \mathrm{dL}$, and 24 -hour urinary albumin excretion $250 \mathrm{mg} /$ day. A renal ultrasound was normal.

Podocyturia was assessed as follows: fresh urine samples were centrifuged at $1500 \mathrm{rpm}$ during $5 \mathrm{~min}$ and the supernatant was discarded; $10 \%$ formaldehyde in phosphatebuffered saline (PBS) $\mathrm{pH}$ 7.2-7.4 was added to the sediment to cover it. Smears were made from each sample on $2 \%$ silane-coated slides. The slides were stained with immunofluorescence technique and observed by epifluorescent microscopy. The slides were preincubated with no immune rabbit serum in phosphate-buffered saline $0.1 \mathrm{M}, \mathrm{pH} 7.4$ (PBS, $1: 100)$ at room temperature for 30 minutes, followed by incubation with a polyclonal anti-synaptopodin antibody (1:100, ab109560 Alexa Fluor ${ }^{\circledR}$, Abcam, Cambridge, United Kingdom) overnight in a wet chamber at $4^{\circ} \mathrm{C}$. After several rinses in PBS, the slides were incubated with anti-rabbit IgG secondary antibody (1:200 Alexa Fluor 488, Abcam, Cambridge, United Kingdom) for 2 hours at room temperature in a wet chamber. Finally, all the slides were mounted using Fluoroshield Mounting Medium with DAPI (Abcam, Cambridge, United Kingdom) and observed in an epifluorescent microscope (Nikon Eclipse E200, Nikon, Tokyo, Japan). Negative controls were performed without primary antibodies. Podocyte counting was assessed by counting in urinary smears the number of cells in 10 microscopy fields of $\times 20$. The podocyte count was 1.07 cells per $\times 20$ field; the number of podocytes per gram of urinary creatinine was 29.9 , and the number of podocytes $/ 100 \mathrm{~mL}$ of urine was 5.35 (Figure 1). This result was compared with 5 controls (Figure 2): 3 males and 2 females; mean age $22 \pm 7.2$ years with no past history of morbidities; creatinine clearance $108 \mathrm{~mL} / \mathrm{min}$; mean 24-hour urinary albumin excretion $88 \pm$ $11 \mathrm{mg} /$ day. The mean podocyte count was $0.12 \pm 0.1$ cells per $\times 20$ field while the mean number of podocytes per gram of urinary creatinine was 10.7 and the mean number of podocytes $/ 100 \mathrm{~mL}$ of urine was 1.1 . The patient declined to undergo a kidney biopsy but accepted to receive amiloride $5 \mathrm{mg}$ /day orally. After three months of therapy new podocyturia and laboratory results were obtained. The podocyte count was 0.2 cells per $\times 20$ field; the number of podocytes per gram of urinary creatinine was 9 , and the number of podocytes $/ 100 \mathrm{~mL}$ of urine was 1 (Figure 3 ). Blood pressure was $110 / 70 \mathrm{mmHg}$, serum creatinine $1.09 \mathrm{mg} / \mathrm{dL}$, creatinine clearance $79 \mathrm{~mL} / \mathrm{min}$, sodium $143 \mathrm{mEq} / \mathrm{L}$, potassium $3.9 \mathrm{mEq} / \mathrm{L}$, and 24-hour urinary albumin excretion $19 \mathrm{mg} /$ day.

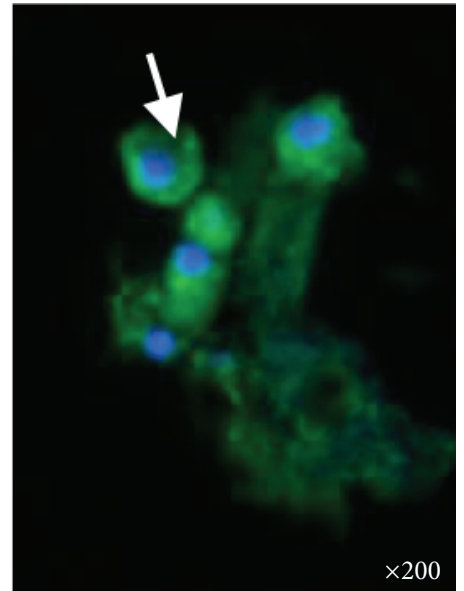

FIGURE 1: White arrow depicts numerous urinary podocytes appearing as bright green round fluorescent cells before amiloride in the patient with Alport syndrome. Fluorescent microscopy $\times 200$.

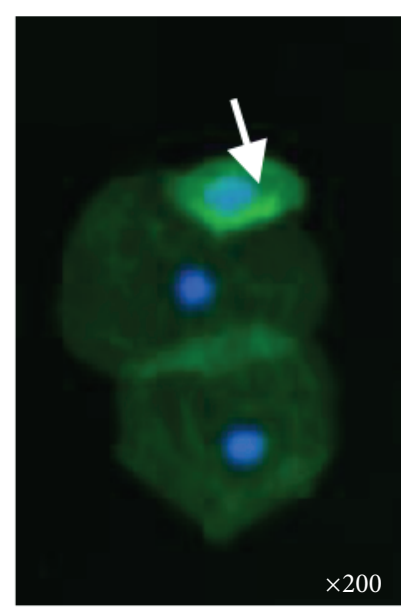

FIGURE 2: Urine specimen from a control. One podocyte (white arrow) and mainly tubular cells are observed. Fluorescent microscopy $\times 200$.

\section{Discussion}

In the present report, we demonstrate that amiloride may prove to be an efficient drug to decrease podocyturia and proteinuria in a patient with chronic kidney disease stage II with Alport syndrome.

Alport syndrome is an inherited progressive form of glomerular disease often associated with sensorineural hearing loss and ocular abnormalities [5-8]. The prevalence of the disease is calculated at approximately 1 in 5,000 live births and accounts for 0.3 to 2.3 percent of new cases of end-stage renal disease [9-11]. Alport syndrome is a primary basement membrane disorder due to mutations in genes encoding several members of type IV collagen. Mutations affecting the $\alpha-3, \alpha-4$, and $\alpha-5$ chains of collagen IV impair their deposition into the collagen network, leading to secondary changes in the glomerular basement membrane composition and to the development of glomerulosclerosis [1]. 


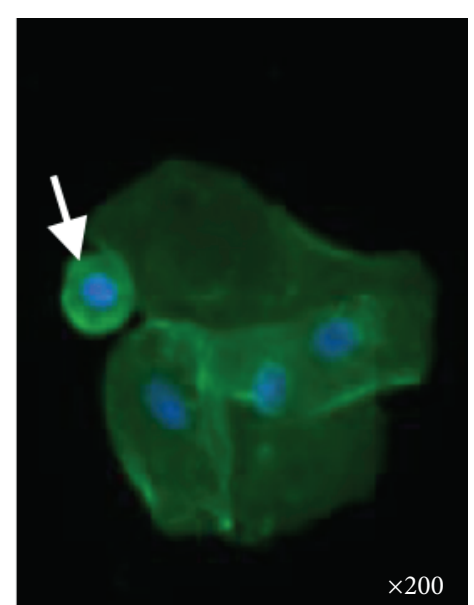

FIGURE 3: Urinary smear displays one podocyte after amiloride (white arrow). Fluorescent microscopy $\times 200$.

The glomerular basement membrane specifically contains collagen type IV, and its structure is severely affected by the abnormal bundles of collagen. Glomerular endothelial cells and podocytes are constitutively attached to the membrane, and all three components function as a histologic and physiologic unit known as the glomerular filtration barrier. Podocytes continuously interact with the basement membrane, creating a permanent cross-talk between the podocyte cellular membrane molecules and the glomerular basement membrane components.

Initially, in Alport disease microhematuria first and proteinuria later on are considered to be mainly due to disruptions in the glomerular basement membrane $[1,2,5-$ 7]. However, proteinuria can also be caused by podocyte depletion in Alport syndrome, in agreement with our findings [12]. Although the molecular mechanisms of podocyte detachment in Alport disease have not been explored, our present report, showing an important reduction in podocyturia after amiloride prescription, may suggest that certain molecules could be involved in podocyte loss. This glomerular podocytopenia is critical as it impairs the normal glomerular filtration process and determines the sclerosis and fibrosis of the glomerulus and the irreversible route to endstage renal disease $[13,14]$.

Plasma and urinary levels of plasminogen and plasmin are elevated in patients with proteinuria and nephrotic syndrome [15]. Plasminogen conversion to plasmin is in part mediated by urokinase-type plasminogen activator (uPA), an enzyme that binds to a specific plasma cell membrane receptor, uPAR [16]. In this regard, amiloride competitively inhibits the catalytic activity of u-PA, decreasing plasmin levels. In turn, plasmin has been implicated in the generation of oedema in glomerulopathies and in the nephrotic syndrome, activating $\mathrm{ENa}^{+} \mathrm{C}$ channels at the distal tubule [17]. Moreover, Zhang et al. have shown that amiloride also reduces $\mathrm{UPAR}$ expression and inhibits uPAR mRNA and protein synthesis in podocytes [18]. UPAR interacts with certain integrins that modulate ligand-binding actions [19]. Lipid raft-associated $\mathrm{UPAR}$ and $\beta_{3}$ integrin, located at the basal compartment of the podocyte anchoring the cell to the basement membrane, form a complex causing the activation of the integrin. It is a key event that mediates uPAR-induced cellular events as actin-mediated podocyte contraction, slit diaphragm widening, increased motility, detachment, and proteinuria [18]. Some studies have also demonstrated the uPAR- $\beta_{3}$ integrin signaling to be involved in the development of proteinuria $[18,20,21]$. We have previously reported a successful enduring antiproteinuric effect mediated by amiloride [22]. Noteworthily, uPAR is highly expressed on the cell surface of diseased podocytes, but only scarcely on normal podocytes [18].

Additionally, plasminogen activator inhibitor-1 (PAI-1) also presents pleiotropic actions, counterbalancing uPA and plasmin actions $[23,24]$. Notably, PAI-1 is not expressed in normal kidney glomeruli but is upregulated in situations of podocyte stress, like uPAR $[18,25]$. PAI-1 interacts with uPAR accelerating podocyturia via the $\alpha_{3} \beta_{1}$ integrin, the main contributor of tight adhesion of podocytes to the glomerular basement membrane, and also through the $\alpha_{v} \beta_{3}$ integrin route $[26,27]$. Therefore, uPAR-activated podocytes via the above-mentioned integrins result in the effacement of foot processes through disruption of the actin cytoskeleton [25]. This mechanism could trigger podocyturia, being potentiated by PAI-1 [25].

Finally, developmental and homeostatic remodeling of the extracellular matrix is a highly regulated process orchestrated by a family of zinc-containing, calcium-dependent, secreted neutral proteases known as the matrix metalloproteinases (MMPs). This family of enzymes can collectively degrade all structural proteins including basement membrane collagens (IV), fibronectin, laminin, proteoglycans, and elastin. The MMPs include collagenases, gelatinases, and atrilysin. Elevated expression of MMP-9 is observed in fibrotic renal cortex from X-linked Alport syndrome dogs. These findings suggest that MMPs may play an important role in matrix accumulation associated with progressive renal scarring in this model [28]. Although not specifically investigated in Alport syndrome, in vitro keratinocyte cell migration towards fibronectin was documented to be stimulated by plasminogen and plasmin by a MMP-9 dependent mechanism and cooperatively interacting with $\beta 1$ integrins. Noteworthily, the addition of the uPA inhibitor amiloride decreased cell migration, probably by decreasing plasmin levels $[17,29]$.

In summary, the decrease in podocyturia displayed by our patient after amiloride administration may suggest that the above-mentioned podocyte molecular pathways may be involved in the pathogenesis of podocyturia and the development of proteinuria in Alport syndrome. Due to the lack of specific treatments, amiloride may prove a useful, nonexpensive drug to be employed in Alport syndrome in order to delay kidney disease progression, mainly by the preservation of the podocyte population.

\section{Conflict of Interests}

No conflict of interests related to the present study is declared by the authors. 


\section{References}

[1] C. E. Kashtan, "Alport syndrome: an inherited disorder of renal, ocular and cochlear basement membranes," Medicine, vol. 78, no. 5, pp. 338-360, 1999.

[2] N. J. A. Webb, S. Shahinfar, T. G. Wells et al., "Losartan and enalapril are comparable in reducing proteinuria in children with Alport syndrome," Pediatric Nephrology, vol. 28, no. 5, pp. 737-743, 2013.

[3] S. U. Vogelmann, W. J. Nelson, B. D. Myers, and K. V. Lemley, "Urinary excretion of viable podocytes in health and renal disease," American Journal of Physiology-Renal Physiology, vol. 285, no. 1, pp. F40-F48, 2003.

[4] H. Trimarchi, "Podocyturia. What is in a name?" Journal of Translational Internal Medicine, vol. 3, pp. 51-56, 2015.

[5] J.-P. Grünfeld, "The clinical spectrum of hereditary nephritis," Kidney International, vol. 27, no. 1, pp. 83-92, 1985.

[6] C. E. Kashtan and A. F. Michael, "Alport syndrome," Kidney International, vol. 50, no. 5, pp. 1445-1463, 1996.

[7] C. E. Kashtan, "Familial hematuria due to type IV collagen mutations: Alport syndrome and thin basement membrane nephropathy," Current Opinion in Pediatrics, vol. 16, no. 2, pp. 177-181, 2004.

[8] L. Massella, A. O. Muda, T. Faraggiana, C. Bette, A. Renieri, and G. Rizzoni, "Epidermal basement membrane $\alpha 5$ (IV) expression in females with Alport syndrome and severity of renal disease," Kidney International, vol. 64, no. 5, pp. 1787-1791, 2003.

[9] M. Levy and J. Feingold, "Estimating prevalence in singlegene kidney diseases progressing to renal failure," Kidney International, vol. 58, no. 3, pp. 925-943, 2000.

[10] U. Persson, J. M. Hertz, J. Wieslander, and M. Segelmark, "Alport syndrome in southern Sweden," Clinical Nephrology, vol. 64, no. 2, pp. 85-90, 2005.

[11] L. Heidet and M.-C. Gubler, "The renal lesions of Alport syndrome," Journal of the American Society of Nephrology, vol. 20, no. 6, pp. 1210-1215, 2009.

[12] L. Wickman, F. Afshinnia, S. Q. Wang et al., "Urine podocyte mRNAs, proteinuria, and progression in human glomerular diseases," Journal of the American Society of Nephrology, vol. 24, no. 12, pp. 2081-2095, 2013.

[13] T. Nakamura, C. Ushiyama, S. Suzuki et al., "Urinary excretion of podocytes in patients with diabetic nephropathy," Nephrology Dialysis Transplantation, vol. 15, no. 9, pp. 1379-1383, 2000.

[14] M. E. Pagtalunan, P. L. Miller, S. Jumping-Eagle et al., "Podocyte loss and progressive glomerular injury in type II diabetes," Journal of Clinical Investigation, vol. 99, no. 2, pp. 342-348, 1997.

[15] N. D. Vaziri, E. C. Gonzales, B. Shayestehfar, and C. H. Barton, "Plasma levels and urinary excretion of fibrinolytic and protease inhibitory proteins in nephrotic syndrome," The Journal of Laboratory and Clinical Medicine, vol. 124, no. 1, pp. 118-124, 1994.

[16] J.-D. Vassalli, D. Baccino, and D. Belin, "A cellular binding site for the Mr 55,000 form of the human plasminogen activator, urokinase," The Journal of Cell Biology, vol.100, no. 1, pp. 86-92, 1985.

[17] H. Trimarchi, "Plasmin, urokinase plasminogen activator receptor and amiloride in the nephrotic syndrome," in Nephrotic Syndrome. Etiology, Pathogenesis and Pathology, M. Mubarak, Ed., Nova Biomedical, New York, NY, USA, 2015.

[18] B. Zhang, S. Xie, W. Shi, and Y. Yang, "Amiloride off-target effect inhibits podocyte urokinase receptor expression and reduces proteinuria," Nephrology, Dialysis, Transplantation, vol. 27, no. 5, pp. 1746-1755, 2012.

[19] H. A. Chapman and Y. Wei, "Protease crosstalk with integrins: the urokinase receptor paradigm," Thrombosis and Haemostasis, vol. 86, no. 1, pp. 124-129, 2001.

[20] C. Wei, C. C. Möller, M. M. Altintas et al., "Modification of kidney barrier function by the urokinase receptor," Nature Medicine, vol. 14, no. 1, pp. 55-63, 2008.

[21] C. Wei, S. El Hindi, J. Li et al., "Circulating urokinase receptor as a cause of focal segmental glomerulosclerosis," Nature Medicine, vol. 17, no. 8, pp. 952-960, 2011.

[22] H. Trimarchi, M. Forrester, F. Lombi et al., "Amiloride as an alternate adjuvant antiproteinuric agent in Fabry disease. The potential roles of plasmin and uPAR," Case Reports in Nephrology, vol. 2014, Article ID 854521, 6 pages, 2014.

[23] C. Dellas and D. J. Loskutoff, "Historical analysis of PAI-1 from its discovery to its potential role in cell motility and disease," Thrombosis and Haemostasis, vol. 93, no. 4, pp. 631-640, 2005.

[24] H. R. Lijnen, "Pleiotropic functions of plasminogen activator inhibitor-1," Journal of Thrombosis and Haemostasis, vol. 3, no. 1, pp. 35-45, 2005.

[25] N. Kobayashi, T. Ueno, K. Ohashi et al., "Podocyte injury-driven intracapillary plasminogen activator inhibitor type 1 accelerates podocyte loss via uPA-mediated $\beta_{1}$-integrin endocytosis," The American Journal of Physiology-Renal Physiology, vol. 308, no. 6, pp. F614-F626, 2015.

[26] M. Regoli and M. Bendayan, "Alterations in the expression of the $\alpha 3 \beta 1$ integrin in certain membrane domains of the glomerular epithelial cells (podocytes) in diabetes mellitus," Diabetologia, vol. 40, no. 1, pp. 15-22, 1997.

[27] N. Sachs and A. Sonnenberg, "Cell-matrix adhesion of podocytes in physiology and disease," Nature Reviews Nephrology, vol. 9, no. 4, pp. 200-210, 2013.

[28] V. H. Rao, G. E. Lees, C. E. Kashtan et al., "Increased expression of MMP-2, MMP-9 (type IV collagenases/gelatinases), and MT1-MMP in canine X-linked Alport syndrome (XLAS)," Kidney International, vol. 63, no. 5, pp. 1736-1748, 2003.

[29] G. J. Thomas, S. Poomsawat, M. P. Lewis, I. R. Hart, P. M. Speight, and J. F. Marshall, " $\alpha \mathrm{v} \beta 6$ integrin upregulates matrix metalloproteinase 9 and promotes migration of normal oral keratinocytes," Journal of Investigative Dermatology, vol. 116, no. 6, pp. 898-904, 2001. 


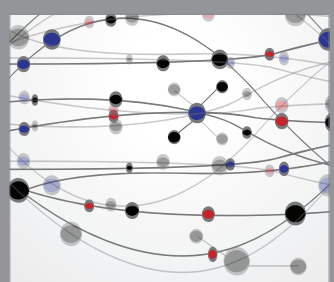

The Scientific World Journal
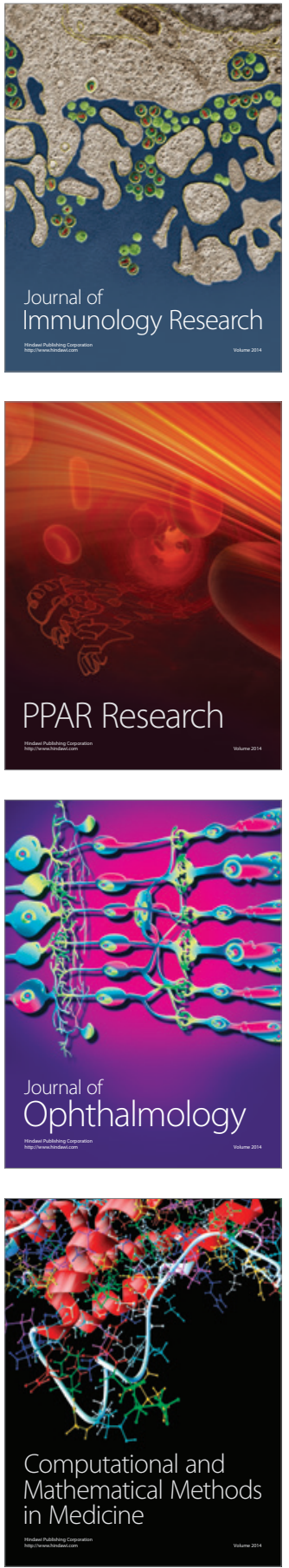

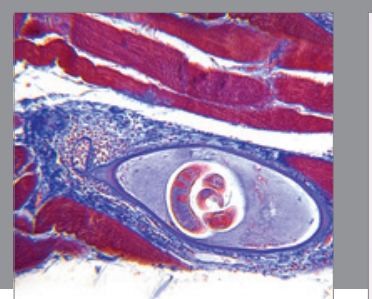

Gastroenterology Research and Practice

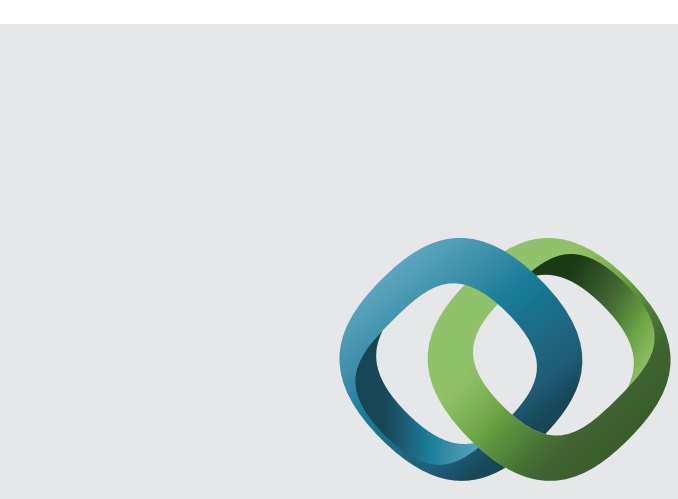

\section{Hindawi}

Submit your manuscripts at

http://www.hindawi.com
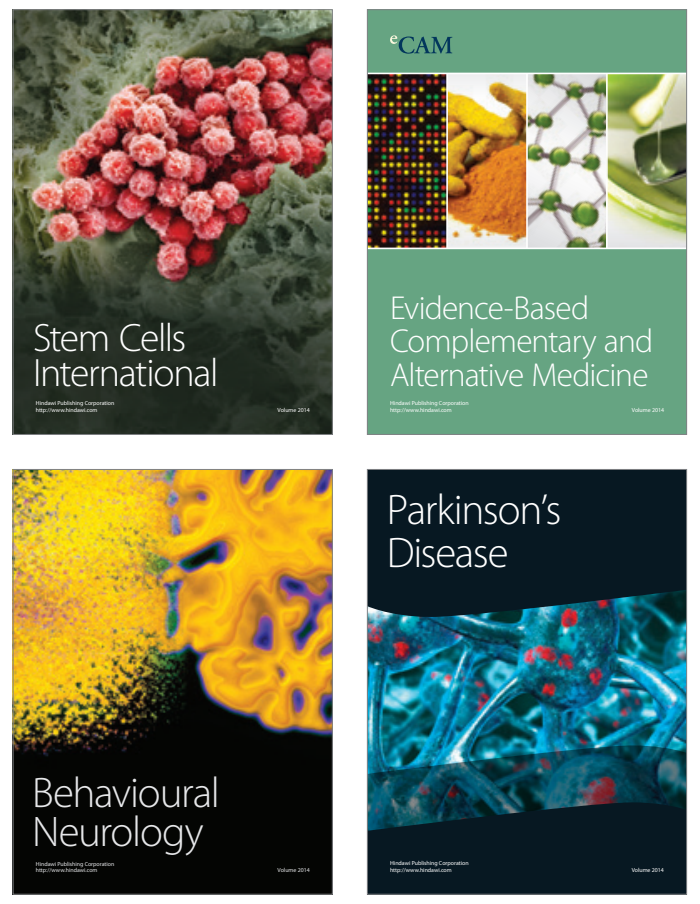
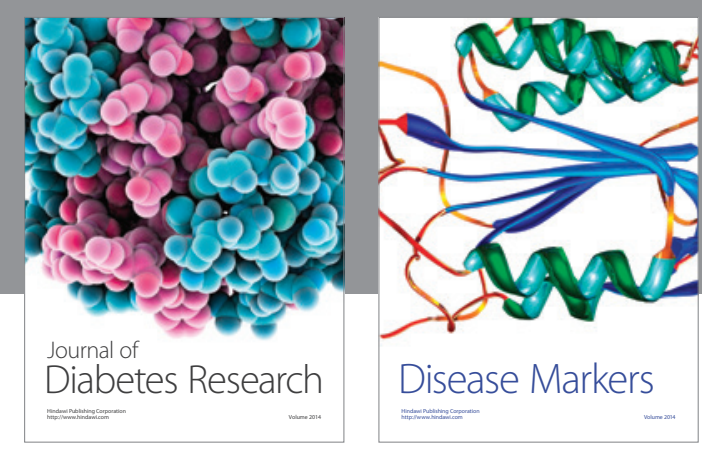

Disease Markers
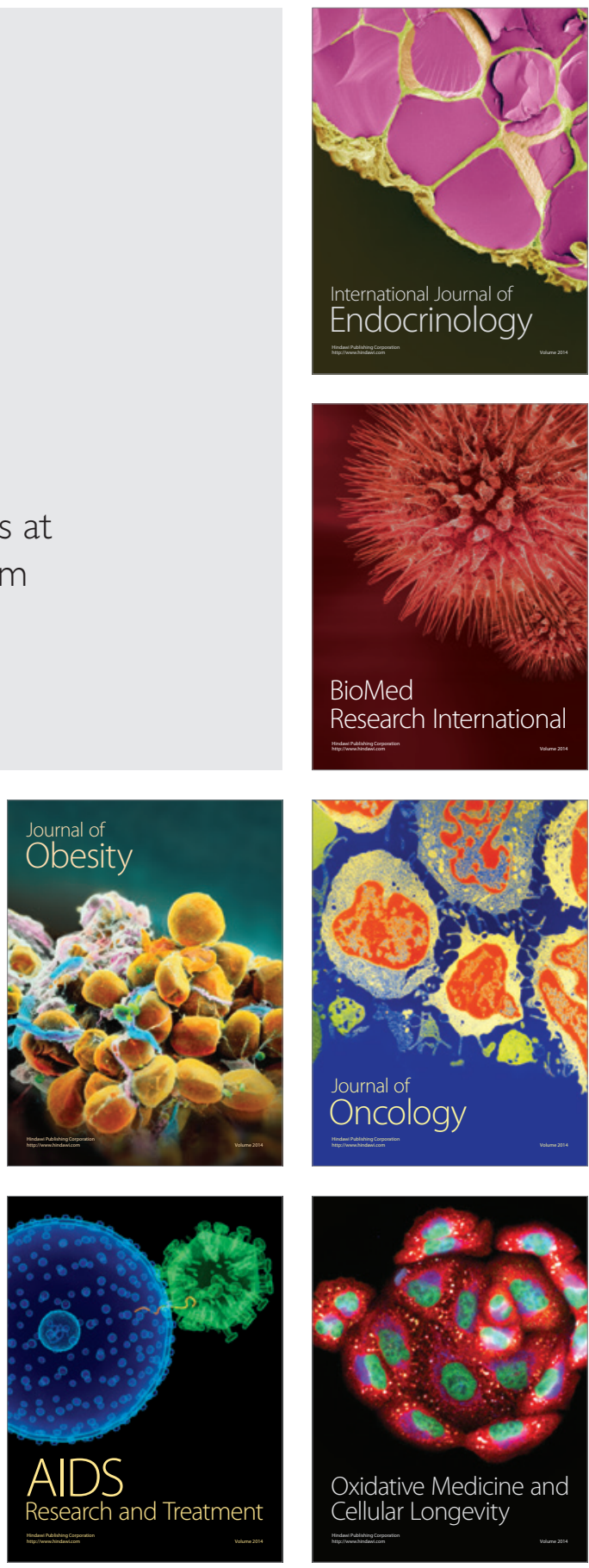